\title{
Influencia de la composición química de diferentes chatarras de acero sobre las propiedades mecánicas de la fundición con grafito esferoidal( ${ }^{\circ}$
}

\author{
I. Asenjo*, P. Larrañaga*, J. Garay* y J. Sertucha*
}

\begin{abstract}
Resumen
Se han seleccionado diferentes chatarras de acero comerciales, con el fin de analizar las implicaciones de su utilización como materias primas y evaluar su aplicabilidad en la fabricación de piezas de fundición con grafito esferoidal. Estos aceros muestran una gran variabilidad en su composición química, dependiendo principalmente de su origen y naturaleza. Se han determinado los elementos químicos aportados por estos materiales y se han estudiado sus efectos sobre las características estructurales y las propiedades mecánicas de piezas test con diferentes morfologías y tamaños. El elemento de aportación más importante es el manganeso, el cual está presente en todos los aceros seleccionados en concentraciones superiores al $0,2 \%$. Otros elementos son: cobre, cromo, estaño, titanio y vanadio. Todos ellos, salvo el titanio y vanadio, originan aumentos en los contenidos de perlita en la matriz metálica, por lo que pueden ser utilizados para producir piezas con estructuras perlíticas y/o ferrítico-perlíticas. En ningún caso se ha detectado la formación de carburos.
\end{abstract}

\section{Influence of the chemical composition of different steel scraps on the mechanical properties of ductile iron}

\begin{abstract}
Different commercial steel scraps have been selected so as to analyse the outcome of their use as raw materials and to evaluate their applicability in the production of ductile iron castings. These selected steels show an important chemical variability mainly depending on their origin and on their previous utilisation. According to that, alloying elements added through these materials have been determined and their effects on the structural characteristics and the mechanical properties of test-castings with different shape and size have been studied. The most important element added is manganese, which is included in all the selected scraps with contents higher than $0.2 \%$. Other elements are: copper, chromium, tin, titanium and vanadium. All of them are known as pearlite promoters apart from titanium and vanadium. Therefore, these elements can be used for manufacturing casting containing pearlitic and/or mixed ferritic and pearlitic structures. No carbides were found in all the test castings.
\end{abstract}

Keywords

Raw materials; Chemical composition; Ductile iron; Mechanical properties; Matrix structure.

\section{INTRODUCCIÓN}

El nivel de desarrollo alcanzado en los procesos de fabricación de piezas de fundición esferoidal implica una mayor competitividad entre las fundiciones por el mercado nacional e internacional de este sector. Por ello, resulta necesario abaratar al máximo los costes de proceso, sin renunciar a la calidad del producto final y/o a la solidez de las metodologías de fabricación. Dentro de las mejoras de competitividad, se plantea la utilización de materias primas alternativas $^{[1]}$, que resulten válidas para la elaboración de la fundición con grafito esferoidal. En este sentido, la incorporación de aceros no convencionales en las cargas metálicas de fusión ${ }^{[2 \text { y } 3]}$ es un campo de estudio con gran interés y aplicación directa en las plantas industriales.

Uno de los aspectos importantes a la hora de desarrollar una metodología optimizada para la fabricación de piezas, es conocer de antemano la cantidad

(•) Trabajo recibido el día de 27 de Julio de 2010 y aceptado en su forma final el día 23 de Febrero de 2011.

* Ingeniería y Procesos de Fundición. Centro de Investigación Metalúrgica AZTERLAN. Aliendalde Auzunea n 6, E-48200. Durango (Bizkaia). E-mail: jsertucha@azterlan.es. 
y tipo de elementos residuales aportados por las materias primas utilizadas. La presencia de muchos de estos elementos en concentraciones minoritarias influye directamente en la germinación y crecimiento del grafito $^{[4-7]}$, condiciona en gran medida la evolución de la matriz metálica durante el proceso de enfriamiento en el estado sólido ${ }^{[8-10]}$, provoca la aparición de fenómenos de segregación durante la solidificación $^{[11 \text { y 12] }}$ y, en muchos casos, favorece la formación de carburos metálicos ${ }^{[13]}$. Por todo ello, la aportación de estos elementos químicos residuales afecta a las características estructurales de las piezas y condiciona sus propiedades mecánicas ${ }^{[8}$ y 13$]$.

Los efectos provocados por la presencia de determinados elementos químicos residuales sobre las fundiciones de hierro han sido objeto de varios estudios. Se ha descrito el efecto estabilizador de carburos y perlitizador del cromo, molibdeno, vanadio y wolframio $^{[6]}$, la influencia del titanio en la maquinabilidad $^{[5]}$, las implicaciones del azufre y manganeso en las propiedades mecánicas ${ }^{[13]}$, el efecto del nitrógeno en la aparición de porosidades de gas ${ }^{[14]}$, la capacidad del magnesio, cerio y lantano para esferoidizar el grafito ${ }^{[4]}$ o del zinc ${ }^{[7]}$, titanio $^{[5]}$ y aluminio ${ }^{[15]}$ para degenerar esta morfología. Todos estos elementos se encuentran presentes en determinados aceros con aplicaciones tecnológicas específicas, los cuales pueden formar parte de componentes reciclados o materiales destinados a su reutilización en la industria de fundición.

La composición química de un determinado acero es, junto con el precio de mercado y la disponibilidad de suministro, el criterio principal para su selección a la hora de formar parte de las cargas metáli$\left.\operatorname{cas}^{[2}{ }^{2} 3\right]$. Teniendo en cuenta la variabilidad en los precios y sus factores de influencia, resulta esencial conocer las características químicas de los aceros disponibles y su posible efecto en las propiedades de la fundición esferoidal. Sin embargo, ésta no es una tarea sencilla. La mayoría de las chatarras de acero disponibles como materias primas se caracterizan por su amplia variedad de procedencias, tipos, tratamien-

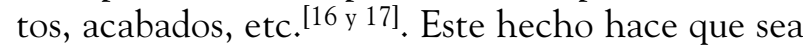
difícil controlar la composición química de estos materiales y, en ocasiones, prácticamente imposible exigir un suministro estable. Un tipo de acero que cumple en cierta medida las exigencias de estabilidad en su composición y disponibilidad es la chapa galvanizada ${ }^{[7]}$, empleada en el sector de automoción. Los requerimientos mecánicos y funcionales en este sector son importantes, por lo que los suministradores de estos aceros deben controlar la regularidad de sus características químicas y físicas. De este modo, los excedentes obtenidos a partir de las operaciones de troquelado o embutición son muy apreciados como materias primas en la industria de la fundición.
La utilización de nuevos materiales en las cargas base, empleadas en los hornos de fusión, lleva necesariamente unido un conocimiento de las posibles aportaciones de elementos no previstos y sus posibles consecuencias sobre las propiedades de las piezas fabricadas. En el presente trabajo, se han seleccionado una serie de chatarras de acero comerciales, con posible aplicación en los procesos de fabricación de piezas de fundición esferoidal, y se han determinado los elementos químicos aportados en cada caso. Los materiales fabricados se han sometido a estudios estructurales, bajo diferentes condiciones de enfriamiento y se han analizado comparativamente sus propiedades mecánicas, con el fin de estimar la viabilidad industrial de los aceros seleccionados como materias primas.

\section{PARTE EXPERIMENTAL}

Se han seleccionado siete tipos de aceros comerciales, procedentes de diversos sectores industriales y con disponibilidad para ser utilizados como materia prima en las cargas de fusión. La tabla I incluye los intervalos de composición química para cada uno de estos materiales. Los valores de composición química se obtuvieron a partir del análisis de cinco muestras tomadas aleatoriamente en cada material. Cada una de estas muestras se fundió en un horno de refundido (SEF-ERSCEM), obteniéndose medallas que fueron analizadas posteriormente utilizando un espectrómetro de emisión por chispa (OBLF QS750).

En la designación empleada en este trabajo para cada tipo de acero, se hace mención directa a su origen. La chatarra de automoción corresponde al paquete galvanizado de uso común en las cargas metálicas en fundición. Este tipo de acero se incluye en el presente estudio como material comparativo con las restantes chatarras. En el caso del acero fragmentado, su origen y composición son menos definidos. Este material está constituido por unidades compactadas de tamaño variable, que contienen trozos de piezas de automoción y otros componentes de acero. La chatarra naval y de raíl corresponde a trozos de acero procedente de los sectores naval y ferroviario, respectivamente. En este último caso, se distinguen dos tipos de material según su contenido en fósforo. La chatarra de alimentación se presenta en forma de paquetes compactados y está constituida por recipientes de acero, destinados al almacenamiento de productos alimenticios. Ocasionalmente, en este material es posible detectar la presencia de algún resto orgánico. Por último, la chatarra de construcción muestra una composición basada principalmente en vigas y otros componentes de acero empleados en este sector. 
Tabla I. Intervalos de composición química para las chatarras de acero seleccionadas (\% en peso)

Table I. Chemical composition ranges of the selected steel scraps (wt. \%)

\begin{tabular}{lcccccc}
\hline Chatarra de acero* $^{*} \mathbf{M n}$ & $\mathbf{P}$ & $\mathbf{T i}$ & $\mathbf{C u}$ & $\mathbf{V}$ & $\mathbf{C r}$ \\
\hline Automoción & $0,2-0,4$ & $0,021-0,037$ & $0,036-0,042$ & $<0,01$ & $0,01-0,1$ & $<0,01$ \\
Fragmentado & $0,2-0,5$ & $0,017-0,022$ & $<0,01$ & $0,2-0,4$ & $<0,01$ & $0,13-0,19$ \\
Naval & $0,8-1,2$ & $0,019-0,024$ & $<0,01$ & $<0,01$ & $<0,01$ & $<0,01$ \\
Raíl & $0,8-1,3$ & $0,025-0,033$ & $<0,01$ & $0,3-0,4$ & $<0,01$ & $0,08-0,12$ \\
Raíl con alto P & $0,8-1,3$ & $0,040-0,082$ & $<0,01$ & $0,3-0,4$ & $<0,01$ & $0,08-0,11$ \\
Alimentación** & $0,2-0,4$ & $0,016-0,019$ & $<0,01$ & $<0,01$ & $<0,01$ & $0,06-0,14$ \\
Construcción & $0,5-1,2$ & $0,018-0,023$ & $0,033-0,078$ & $0,2-0,6$ & $<0,01$ & $0,10-0,22$ \\
\hline
\end{tabular}

* El contenido de azufre en estos materiales se encuentra en el intervalo 0,015-0,035\%.

${ }^{* *}$ El contenido de estaño se estima en el intervalo 0,20 - 0,25\%.
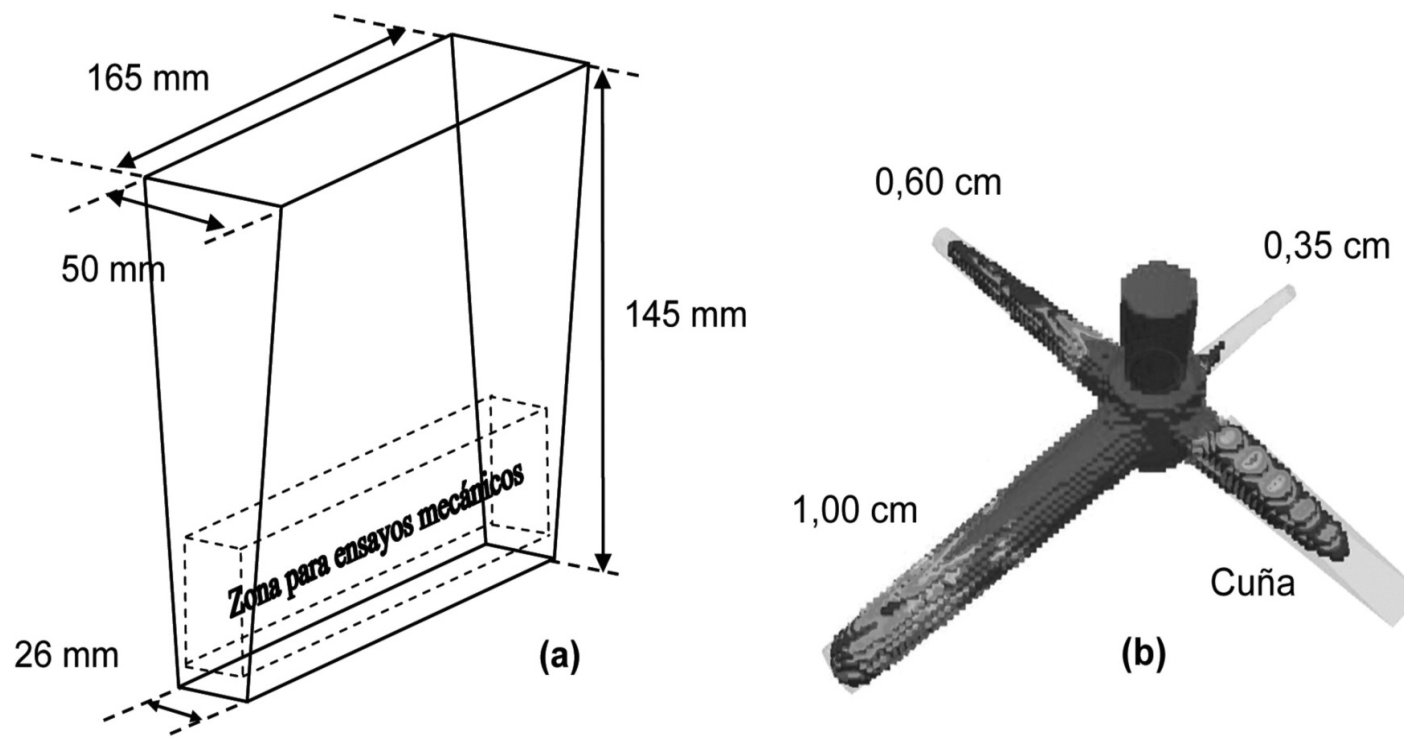

(b)
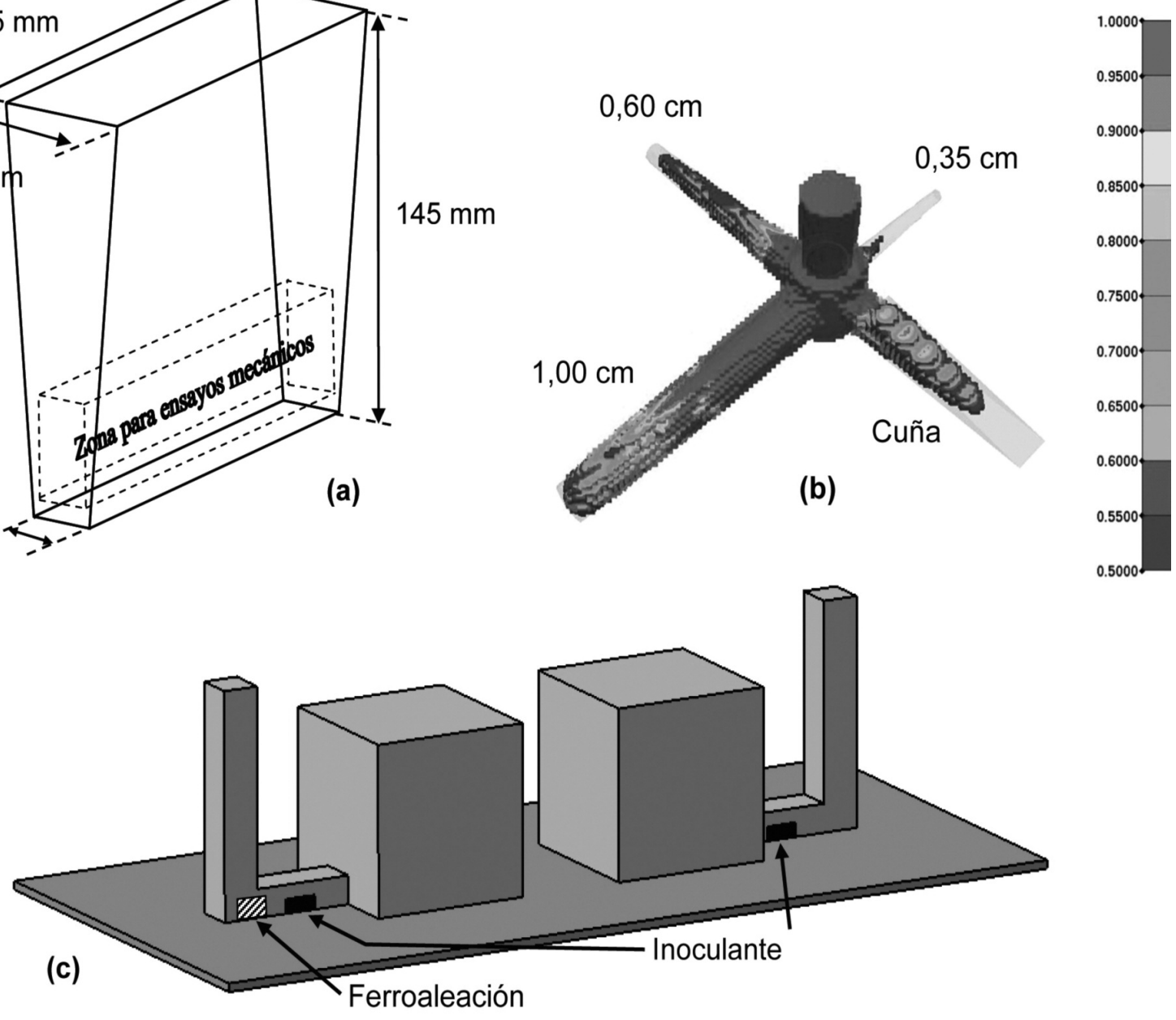

Figura 1. Piezas test fabricadas: a) probeta normalizada; b) piezas cilíndricas y cuña; c) bloques cúbicos.

Figure 1. Test castings manufactured in this work: a) standard keel-block; b) cylindrical castings and wedge casting; c) cubic blocks. 
Las piezas test utilizadas en este trabajo para la caracterización de las aleaciones fabricadas son las siguientes:

- Una probeta normalizada tipo Y2, según UNE-EN-1563 (Fig. 1 a)).

- Tres piezas cilíndricas con módulos geométri$\cos 0,35 ; 0,60$ y $1,00 \mathrm{~cm}$ (Fig. 1 b)) y una pieza en forma de cuña destinada al estudio de la formación de carburos en las secciones con menor espesor (Fig. 1 b)).

- Bloques cúbicos de $300 \mathrm{~mm}$ de lado y un módulo geométrico de $5,00 \mathrm{~cm}$ (Fig. $1 \mathrm{c})$ ). Este tipo de pieza test, se ha empleado para estudiar la influencia de los elementos de aportación más importantes, en las características de aquellos materiales sometidos a enfriamientos lentos.

Todas estas piezas test se fabricaron en moldes de arena aglomerada químicamente. El proceso de inoculación se llevó a cabo en el interior de los moldes, adicionando el 0,20 \% del peso de metal colado, en forma de un inoculante comercial (\% en peso $\mathrm{Si}=77,1 ; \mathrm{Al}=3,9 ; \mathrm{Ca}=1,3 ; \mathrm{TR}=0,5$ y Fe = 17,2). Los moldes utilizados en la fabricación de los bloques cúbicos contenían dos huellas (Fig. 1 c)), con el fin de comparar las características de las aleaciones con y sin la adición de los elementos químicos seleccionados para fabricar este tipo de pieza test (manganeso, cromo y vanadio). Para ello, en cada caso, se fijaron con ayuda de un adhesivo las cantidades específicas de la correspondiente ferroaleación, en el canal de llenado perteneciente a una de las huellas contenidas en el molde. Estas adiciones fueron: $400 \mathrm{~g}$ de FeMn (\% en peso $\mathrm{Mn}=75,4$ y $\mathrm{Fe}=24,6$ ), $340 \mathrm{~g}$ de $\mathrm{FeCr}(\%$ en peso $\mathrm{Cr}=70,1$ y $\mathrm{Fe}=29,9)$ ó $175 \mathrm{~g}$ de $\mathrm{FeV}$ ( $\%$ en peso $\mathrm{V}=71,6$ y $\mathrm{Fe}=28,4$ ).

En el caso de las piezas test con módulos geométricos comparativamente más bajos (probetas normalizadas, piezas cilíndricas y con forma de cuña), se prepararon cargas metálicas utilizando cada una de las chatarras de acero incluidas en la tabla I. Estas cargas estuvieron constituidas por $25 \mathrm{~kg}$ del correspondiente acero, $25 \mathrm{~kg}$ de retornos procedentes de la fabricación de piezas de fundición esferoidal de matriz ferrítica (\% en peso $\mathrm{C}=3,81 ; \mathrm{Si}=2,54 ; \mathrm{Mn}=0,12 ; \mathrm{P}=0,034$; $\mathrm{Cu}=0,02 ; \mathrm{Ti}=0,014 ; \mathrm{Cr}=0,033$ y Fe =93,4), 1,2 kg de un recarburante de grafito y $0,5 \mathrm{~kg}$ de $\mathrm{FeSi}$ (\% en peso $\mathrm{Si}=74,8 ; \mathrm{Al}=1,5$ y $\mathrm{Fe}=23,7$ ). Estos materiales se introdujeron en un horno de inducción de media frecuencia $(250 \mathrm{~Hz})$, con capacidad máxima para $100 \mathrm{~kg}$ y $100 \mathrm{kw}$ de potencia. En el caso de los bloques cúbicos de elevado módulo geométrico, se prepararon tres cargas metálicas constituidas por $2.500 \mathrm{~kg}$ de lingote, $2.500 \mathrm{~kg}$ de paquete de automoción y $5.000 \mathrm{~kg}$ de retornos de baja aleación (mazarotas, canales de llenado y copas de colada utilizados en la fabricación de piezas ferríticas destinadas al sector eólico). Estas cargas, junto con las correspondientes ferroaleaciones, se introdujeron en un horno de media frecuencia $(250 \mathrm{~Hz})$, con capacidad para 12 t y $500 \mathrm{kw}$ de potencia. Tras la fusión de las cargas, se obtuvieron medallas de composición química y se realizaron correcciones de los contenidos de carbono y silicio, con el fin de alcanzar contenidos similares de estos dos elementos en todos los casos. La tabla II, muestra las composiciones químicas finales obtenidas en todos los metales base fabricados. Los contenidos de carbono y azufre, se determinaron con ayuda de un equipo Leco CS 244. Para el resto de elementos, se analizaron las correspondientes medallas, con un espectrómetro de emisión por chispa (OBLF QS750).

Los tratamientos de nodulización se efectuaron empleando una cuchara de $50 \mathrm{~kg}$, cuando el metal base procedía del horno de fusión con capacidad para $100 \mathrm{~kg}$, ó una cuchara de $2.500 \mathrm{~kg}$, cuando el origen del metal base era el horno de fusión, con capacidad para 12 t (bloques cúbicos).

Todos los tratamientos de nodulización se realizaron siguiendo la metodología "sandwich".

Para ello, en la cámara de reacción de las cucharas de $50 \mathrm{~kg}$, se introdujo el 1,2\% $(0,6 \mathrm{~kg})$ de la ferroaleación $\mathrm{FeSiMg} 511(\% \mathrm{Si}=44,7 ; \% \mathrm{Mg}=5,6$; $\% \mathrm{Ca}=1,2 ; \% \mathrm{TR}=0,7$ y $\mathrm{Fe}=47,8)$ antes del vertido del metal en su interior. En el caso de las cucharas de $2.500 \mathrm{~kg}$, en la cámara de reacción se introdujo el 0,8\% (20 kg) de esta misma ferroaleación. El material cubriente utilizado estaba constituido por recortes de acero (tamaño $5-15 \mathrm{~mm}$ ), procedentes de procesos de estampación. El traspaso del metal base a las cucharas de tratamiento se efectuó cuando la temperatura de éste se encontraba en el intervalo $1.480-1.500{ }^{\circ} \mathrm{C}$ (cuchara de $50 \mathrm{~kg}$ ) y $1.400-1.420^{\circ} \mathrm{C}$ (cuchara de $2.500 \mathrm{~kg}$ ). Al finalizar la reacción del tratamiento con magnesio, el desescoriado del metal se realizó en la propia cuchara, se obtuvo una medalla para analizar su composición química y se colaron finalmente los moldes.

El estudio de las propiedades mecánicas de todas las aleaciones fabricadas se llevó a cabo utilizando probetas de tracción de $10 \mathrm{~mm}$ de diámetro, mecanizadas a partir de la parte inferior de las probetas normalizadas tipo Y2 (Fig. 1 a)) y la zona central de las piezas cilíndricas y los bloques cúbicos fabricados. Tras efectuar los ensayos de tracción, las probetas se utilizaron para determinar la dureza Brinell de los materiales, utilizando una esfera de $10 \mathrm{~mm}$ de diámetro y una carga de $3.000 \mathrm{~kg}$. Los ensayos de dureza se efectuaron en las zonas no afectadas por el ensayo de tracción. Finalmente, estas muestras fueron cortadas y preparadas convenientemente para llevar a cabo las caracterizaciones metalográficas. Se analizó 
INFLUENCIA DE LA COMPOSICIÓN QUIMIICA DE DIFERENTES CHATARRAS DE ACERO SOBRE LAS PROPIEDADES MECÁNICAS DE LA FUNDICIÓN CON GRAFITO ESFEROIDAL INFLUENCE OF THE CHEMICAL COMPOSITION OF DIFFERENT STEEL SCRAPS ON THE MECHANICAL PROPERTIES OF DUCTILE IRON

Tabla II. Composición química de los metales base preparados (\% en peso)

Table II. Chemical composition of the base melts prepared in this work (wt. \%)

\begin{tabular}{lcccccccccc}
\hline & \multicolumn{8}{c}{ Probeta normalizada, piezas cilíndricas y cuña } \\
\hline Metal base & $\mathbf{C}$ & $\mathbf{S i}$ & $\mathbf{M n}$ & $\mathbf{P}$ & $\mathbf{S}$ & $\mathbf{C u}$ & $\mathbf{T i}$ & $\mathbf{C r}$ & $\mathbf{S n}$ \\
\hline Automoción & 3,94 & 2,18 & 0,28 & 0,029 & 0,011 & 0,07 & 0,025 & 0,038 & $<0,005$ \\
Fragmentado & 3,90 & 2,13 & 0,29 & 0,024 & 0,013 & 0,10 & 0,018 & 0,087 & $<0,005$ \\
Naval & 3,95 & 1,95 & 0,70 & 0,022 & 0,009 & 0,07 & 0,014 & 0,033 & $<0,005$ \\
Raíl & 3,91 & 2,12 & 0,61 & 0,031 & 0,014 & 0,18 & 0,013 & 0,053 & $<0,005$ \\
Raíl con alto P & 3,98 & 2,14 & 0,66 & 0,044 & 0,010 & 0,21 & 0,012 & 0,055 & $<0,005$ \\
Alimentación & 3,92 & 1,99 & 0,18 & 0,025 & 0,012 & 0,06 & 0,015 & 0,060 & 0,13 \\
Construcción & 3,92 & 2,05 & 0,40 & 0,022 & 0,010 & 0,12 & 0,033 & 0,080 & $<0,005$ \\
& & & & & Bloques cúbicos & & & \\
\hline & & $\mathbf{C}$ & $\mathbf{S i}$ & $\mathbf{M n}$ & $\mathbf{P}$ & $\mathbf{S}$ & $\mathbf{C u}$ & $\mathbf{T i}$ & $\mathbf{C r}$ & $\mathbf{V}$ \\
\hline Metal base & 3,89 & 1,57 & 0,16 & 0,026 & 0,018 & 0,03 & 0,015 & 0,03 & 0.009 \\
\hline Automoción 1 & 3,85 & 1,54 & 0,13 & 0,024 & 0,019 & 0,02 & 0,012 & 0,02 & 0,008 \\
Automoción 2 & 3,88 & 1,55 & 0,18 & 0,036 & 0,017 & 0,02 & 0,011 & 0,02 & 0,008 \\
Automoción 3 & & & & & & & & & \\
\hline
\end{tabular}

el índice de esferoidización de los grafitos, la cantidad de éstos por unidad de superficie $(\mathrm{N})$ y el tipo de constituyentes estructurales.

La distribución de estas fases se determina en cuatro campos metalográficos diferentes, con ayuda de un software específico para el análisis de imágenes. Para llevar a cabo la caracterización microestructural, las superficies de inspección se atacan con el reactivo Nital 5 durante un período de $10-15 \mathrm{~s}$.

\section{RESULTADOS Y DISCUSIÓN}

La tabla III incluye los resultados de los análisis de composición química, realizados sobre todos los metales de colada preparados en el presente trabajo. Dado que las adiciones de FeMn, FeCr y FeV en los bloques cúbicos se efectuaron directamente en el interior de los moldes, sus composiciones químicas se han determinado directamente sobre pieza. En el resto de los casos, los análisis químicos se efectuaron sobre las probetas normalizadas tipo Y2. Los contenidos de carbono y silicio en los metales de colada son similares, con el fin de minimizar la influencia de las variaciones en el carbono equivalente sobre las características estructurales de los materiales fabricados. En el caso de los bloques cúbicos, el carbono equivalente es inferior al resto de piezas test $(4,40-4,54 \%)$, de acuerdo con los criterios de composición empleados habitualmente en la fabricación de piezas de gran tamaño con importantes exigencias en sus resistencias al impacto.

$\mathrm{Al}$ analizar comparativamente los valores mostrados en las tablas II y III, se comprueba que el manganeso es el principal elemento aportado por los diferentes aceros seleccionados en este estudio. Por otra parte, la reacción producida por el tratamiento del metal con FeSiMg no origina variaciones en los principales elementos aportados por las chatarras empleadas, es decir, los contenidos de manganeso, fósforo, cromo, titanio, estaño y cobre son similares a los detectados en los correspondientes metales base (Tabla II). Este aspecto debe ser tenido en cuenta de cara a evaluar la utilización de estos aceros en la fabricación industrial de piezas ferríticas, tal y como se discutirá al final del apartado. Otro elemento a tener en cuenta debido a su capacidad para originar porosidades de gas durante la solidificación es el nitrógeno ${ }^{[14]}$. Los contenidos de este elemento en el metal de colada se sitúan en el intervalo 40 - 58 ppm en todos los casos, excepto en las aleaciones preparadas con el acero de automoción, donde se obtiene una concentración ligeramente superior (55 - $77 \mathrm{ppm})$. En cualquier caso, estos contenidos pueden considerarse habituales en la fabricación de piezas de fundición esferoidal, por lo que no suponen en sí una amenaza a la hora de originar este tipo de porosidades ${ }^{[14]}$.

La mayoría de los elementos de aleación aportados por los aceros utilizados en la preparación del metal 
Tabla III. Composición química de los metales tratados con Mg (\% en peso)

Table III. Chemical composition of the Mg-treated melts (wt. \%)

\begin{tabular}{|c|c|c|c|c|c|c|c|c|c|c|}
\hline \multirow[b]{2}{*}{ Metal tratado } & \multicolumn{10}{|c|}{ Probeta normalizada, piezas cilíndricas y cuña } \\
\hline & C & Si & Mn & $\mathbf{P}$ & $\mathbf{S}$ & $\mathrm{Cu}$ & $\mathrm{Ti}$ & $\mathbf{M g}$ & $\mathrm{Cr}$ & Sn \\
\hline Automoción & 3,74 & 2,73 & 0,30 & 0,025 & 0,012 & 0,10 & 0,024 & 0,051 & 0,036 & $<0,005$ \\
\hline Fragmentado & 3,75 & 2,66 & 0,29 & 0,024 & 0,010 & 0,07 & 0,017 & 0,040 & 0,088 & $<0,005$ \\
\hline Naval & 3,77 & 2,59 & 0,73 & 0,022 & 0,008 & 0,07 & 0,019 & 0,051 & 0,033 & $<0,005$ \\
\hline Raíl & 3,77 & 2,68 & 0,60 & 0,030 & 0,012 & 0,18 & 0,018 & 0,045 & 0,053 & $<0,005$ \\
\hline Raíl con alto $P$ & 3,66 & 2,62 & 0,65 & 0,045 & 0,012 & 0,20 & 0,015 & 0,041 & 0,056 & $<0,005$ \\
\hline Alimentación & 3,68 & 2,64 & 0,19 & 0,023 & 0,008 & 0,07 & 0,014 & 0,035 & 0,063 & 0,12 \\
\hline \multirow[t]{2}{*}{ Construcción } & 3,80 & 2,58 & 0,40 & 0,021 & 0,006 & 0,12 & 0,034 & 0,051 & 0,082 & $<0,005$ \\
\hline & \multicolumn{10}{|c|}{ Bloques cúbicos } \\
\hline Metal tratado & C & Si & Mn & $\mathbf{P}$ & S & $\mathrm{Cu}$ & $\mathrm{Ti}$ & $\mathbf{M g}$ & $\mathrm{Cr}$ & V \\
\hline Automoción 1 & 3,78 & 2,21 & 0,16 & 0,033 & 0,010 & 0,01 & 0,013 & 0,048 & 0,03 & 0,007 \\
\hline Automoción 1 - Mn & 3,81 & 2,18 & 0,31 & 0,035 & 0,014 & 0,02 & 0,010 & 0,039 & 0,03 & 0,009 \\
\hline Automoción 2 & 3,73 & 2,12 & 0,12 & 0,029 & 0,012 & 0,01 & 0,011 & 0,038 & 0,02 & 0,008 \\
\hline Automoción 2 - Cr & 3,71 & 2,14 & 0,13 & 0,031 & 0,012 & 0,01 & 0,012 & 0,042 & 0,11 & 0,009 \\
\hline Automoción 3 & 3,77 & 2,15 & 0,18 & 0,030 & 0,015 & 0,01 & 0,010 & 0,034 & 0,02 & 0,006 \\
\hline Automoción 3 - V & 3,76 & 2,15 & 0,18 & 0,033 & 0,015 & 0,01 & 0,009 & 0,038 & 0,02 & 0,059 \\
\hline
\end{tabular}

base están considerados como promotores de perlita. Algunos de ellos, se emplean industrialmente para originar esta fase estructural en las piezas (manganeso, cobre y estaño). Adicionalmente, determinados elementos también son capaces de segregar hacia las últimas áreas en solidificar y originar carburos en estas zonas (manganeso, vanadio y cromo). En la tabla IV, se muestran de forma comparativa los resultados de los estudios metalográficos realizados sobre todas las piezas test fabricadas en este trabajo. En la figura 2 se muestran las estructuras matriciales pertenecientes a la probeta normalizada, y las tres piezas test fabricadas con el acero fragmentado, además de las correspondientes a los bloques cúbicos con y sin adición de FeMn. No se ha detectado la presencia de carburos en estas piezas, a excepción de las cuñas fabricadas con el fin de evaluar la capacidad del metal para formar esta fase bajo las condiciones de enfriamiento más rápidas (Fig. 1 b)). La tabla V, contiene los valores de la altura ocupada por los carburos en la sección triangular de las mencionadas cuñas.

Los dos parámetros que muestran una mayor influencia sobre las características estructurales de las piezas test son la velocidad de enfriamiento del material (módulo de enfriamiento) y el contenido de los elementos aportados. En la tabla IV, se observa que la densidad de esferoides se encuentra fuertemente influida por el tamaño de la pieza fabricada, es decir, por su velocidad de solidificación. Como era de esperar, un aumento de este último parámetro origina una mayor densidad de esferoides grafíticos en el material. Sin embargo, no se ha encontrado relación alguna entre la densidad de esferoides y la presencia de los elementos de aleación aportados. En este punto, es necesario recordar las precauciones tomadas a la hora de trabajar con carbonos equivalentes y condiciones de inoculación similares, con el fin de minimizar posibles diferencias en la influencia de estas dos variables sobre las características estructurales de las piezas test y/o los bloques cúbicos ${ }^{[18]}$.

Por otro lado, tanto la velocidad de enfriamiento como el contenido de los elementos de aleación presentes en el metal, ejercen una gran influencia sobre el tipo de estructura matricial obtenido. La cantidad de perlita formada aumenta al reducir la sección de las piezas y al incrementar el contenido de los elementos promotores de esta fase estructural (manganeso, cobre, cromo y estaño). El efecto de la velocidad de enfriamiento puede ser fácilmente comprobado, comparando las estructuras matriciales de las tres piezas cilíndricas (Fig. 1 b)), fabricadas con un mismo metal. Al analizar los resultados obtenidos a partir de las diferentes 
Tabla IV. Índice de esferoidización (IE), densidad de esferoides (N) y estructura matricial obtenidos a partir de los análisis metalográficos

Table IV. Nodularity, nodule count $(N)$ and structure type obtained from the metallographic analysis

\begin{tabular}{|c|c|c|c|c|}
\hline Carga metálica & Pieza & IE (\%) & $\mathbf{N}\left(\mathrm{mm}^{-2}\right)$ & $F / P(\%)$ \\
\hline Automoción & & 90 & 233 & $85 / 15$ \\
\hline Fragmentado & & $>90$ & 204 & $60 / 40$ \\
\hline Naval & & $>90$ & 228 & $45 / 55$ \\
\hline Raíl & Probeta & $>90$ & 226 & $50 / 50$ \\
\hline Raíl con alto $\mathrm{P}$ & normalizada & 90 & 244 & $45 / 55$ \\
\hline Alimentación & & $>90$ & 235 & $5 / 95$ \\
\hline Construcción & & $>90$ & 226 & $55 / 45$ \\
\hline Automoción & & $>90$ & 697 & $80 / 20$ \\
\hline Fragmentado & & $>90$ & 590 & $50 / 50$ \\
\hline Naval & & $>90$ & 628 & $35 / 65$ \\
\hline Raíl & Pieza cilíndrica & $>90$ & 680 & $45 / 55$ \\
\hline Raíl con alto $\mathrm{P}$ & Módulo 0,35 cm & $>90$ & 692 & $40 / 60$ \\
\hline Alimentación & & $>90$ & 636 & $5 / 95$ \\
\hline Construcción & & $>90$ & 706 & $45 / 55$ \\
\hline Automoción & & 90 & 552 & $90 / 10$ \\
\hline Fragmentado & & $>90$ & 522 & $60 / 40$ \\
\hline Naval & & $>90$ & 535 & $45 / 55$ \\
\hline Raíl & Pieza cilíndrica & $>90$ & 556 & $50 / 50$ \\
\hline Raíl con alto $\mathrm{P}$ & Módulo 0,60 cm & $>90$ & 554 & $50 / 50$ \\
\hline Alimentación & & $>90$ & 486 & $10 / 90$ \\
\hline Construcción & & $>90$ & 537 & $55 / 45$ \\
\hline Automoción & & 90 & 446 & $92 / 8$ \\
\hline Fragmentado & & $>90$ & 393 & $70 / 30$ \\
\hline Naval & Pieza cilíndrica & $>90$ & 441 & $55 / 45$ \\
\hline Raíl & Módulo 1,00 cm & $>90$ & 481 & $55 / 45$ \\
\hline Raíl con alto $\mathrm{P}$ & & $>90$ & 451 & $50 / 50$ \\
\hline Alimentación & & $>90$ & 472 & $15 / 85$ \\
\hline Construcción & & $>90$ & 392 & $65 / 35$ \\
\hline Automoción 1 & & $>90$ & 120 & $98 / 2$ \\
\hline Automoción 1 - Mn & & $>90$ & 125 & $65 / 35$ \\
\hline Automoción 2 & Bloques cúbicos & $>90$ & 128 & $98 / 2$ \\
\hline Automoción 2 - Cr & & $>90$ & 139 & $90 / 10$ \\
\hline Automoción 3 & & $>90$ & 135 & $95 / 5$ \\
\hline Automoción 3 - V & & $>90$ & 118 & $95 / 5$ \\
\hline
\end{tabular}

aleaciones, se observa que el cobre y especialmente el estaño son los perlitizantes más potentes.

El efecto perlitizador de estos dos elementos ha sido descrito anteriormente en la bibliografía ${ }^{[9,19}$ y 20$]$. Sin embargo, también es necesario tener en cuenta el importante efecto perlitizador del manganeso ${ }^{[9}$ y 20$]$, puesto que se trata del principal elemento de aportación de las chatarras seleccionadas en este trabajo. En el caso de los bloques cúbicos, la adición de manganeso también origina un aumento importante del contenido de perlita (Tabla IV), a pesar de que la velocidad de enfriamiento es lenta.

La concentración máxima de cromo en las probetas normalizadas y las piezas cilíndricas no alcanza el 0,09\%, cuando se emplean los dos aceros que tienen mayores contenidos de este elemento (fragmen- 


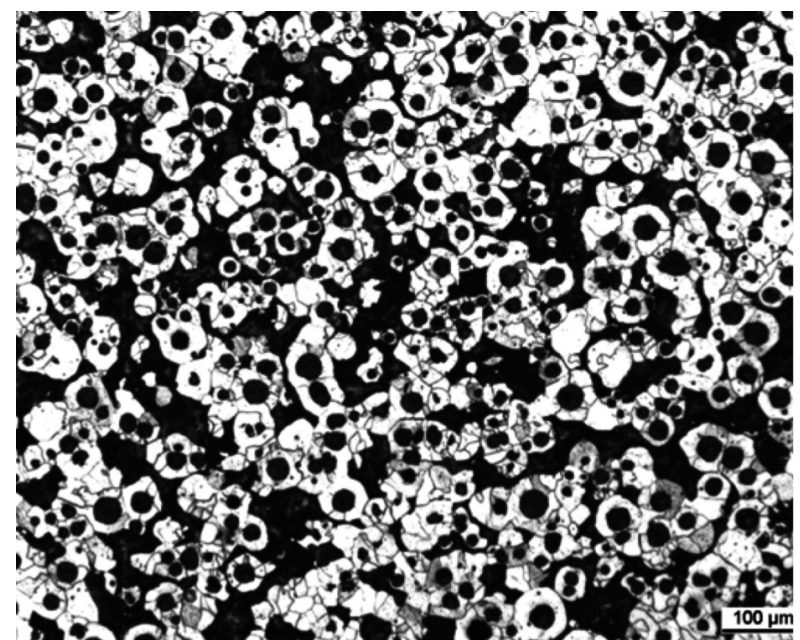

(a)

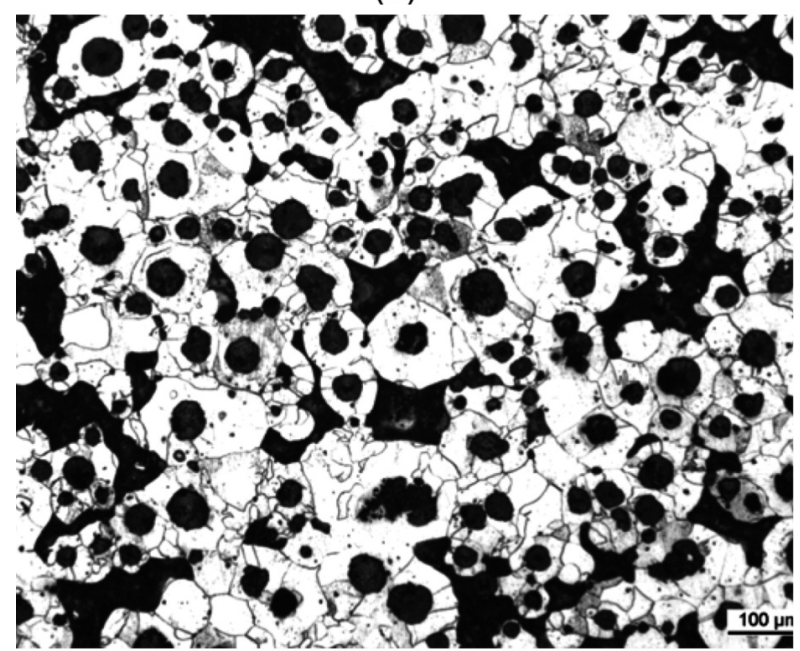

(c)

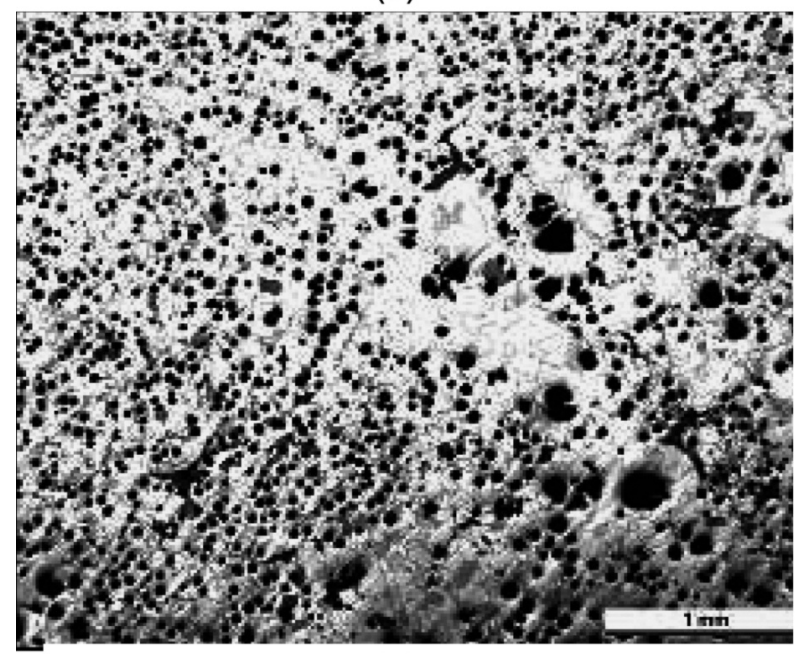

(e)

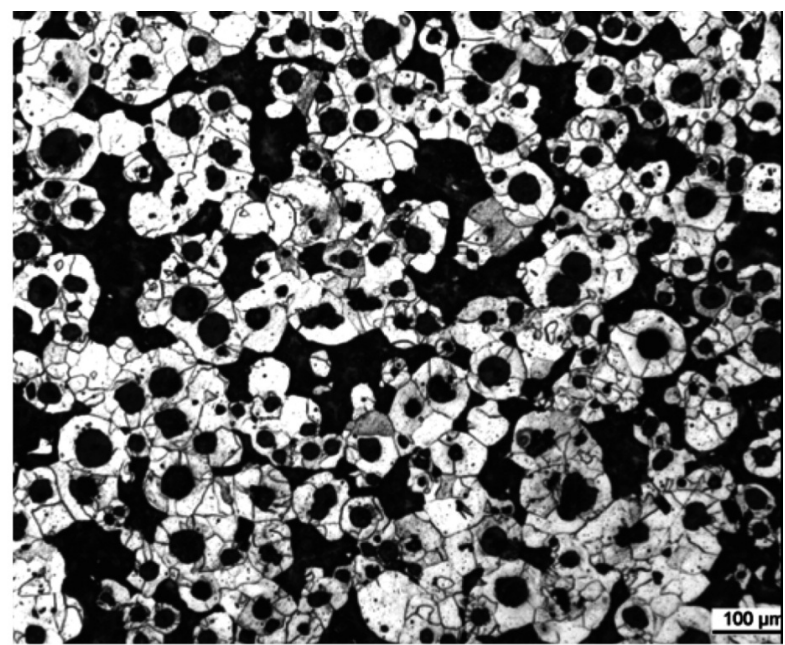

(b)

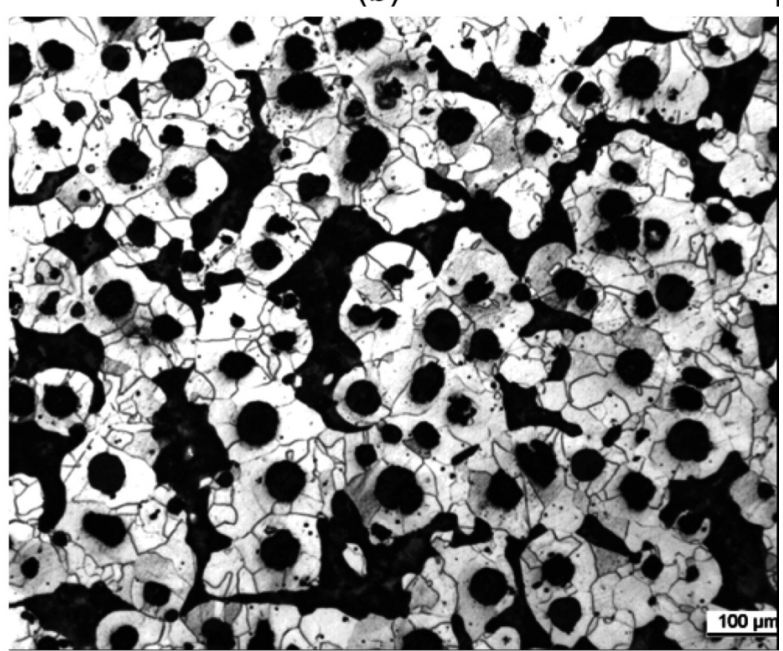

(d)

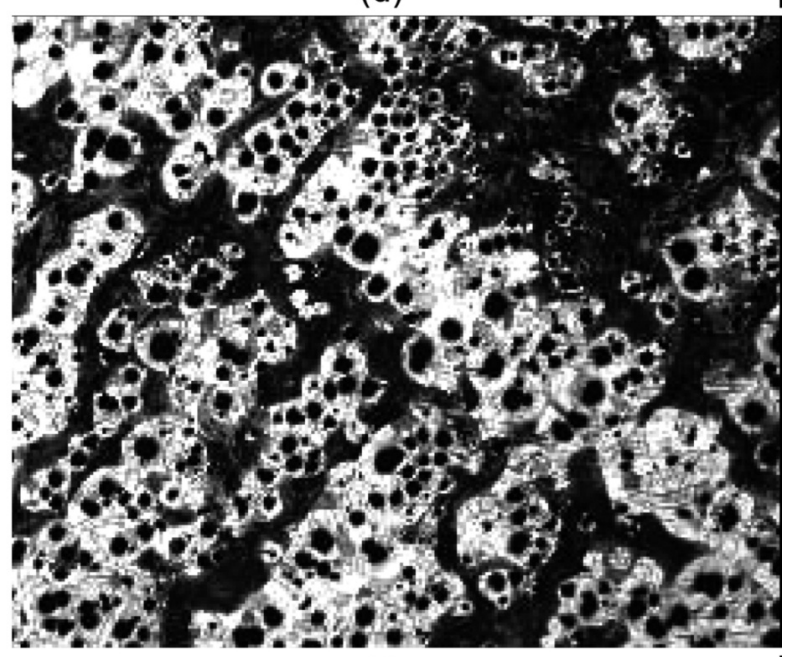

(f)

Figura 2. Estructura matricial en: a) pieza test $M=0,35 \mathrm{~cm}$; b) pieza test $M=0,60 \mathrm{~cm}$; ) pieza test $\mathrm{M}=1,00 \mathrm{~cm} ; \mathrm{d}$ ) probeta normalizada (fabricadas con acero fragmentado); e) bloque cúbico Automoción1 y f) bloque cúbico Automoción1 - Mn.

Figure 2. Matrix structure of: a) test casting $M=0.35 \mathrm{~cm}$; b) test casting $M=0.60 \mathrm{~cm}$; ) test casting $M=1.00 \mathrm{~cm} ; d$ ) standard keel-block (all manufactured using fragmented steel; e) cubic block Automoción1 and f) cubic block Automoción1 - Mn. 
tado y de construcción). Debido a esto y al hecho de que existen otros elementos de aporte en estas chatarras, el efecto perlitizador del cromo sólo puede ser observado claramente en la fabricación del bloque cúbico con adición de este elemento (Tabla III).

Un caso similar al cromo se obtiene para el vanadio. Aunque este elemento puede aparecer principalmente en las chatarras de automoción, la concentración máxima obtenida en el metal de colada es 90 ppm cuando se utiliza este tipo de acero en la carga metálica. De este modo, sólo ha sido posible analizar los efectos del vanadio, a través de su adición en el correspondiente bloque cúbico (Tabla III). En este caso, no se ha detectado efecto alguno del vanadio sobre las características analizadas metalográficamente (Tabla IV).

En el caso del titanio, las aportaciones en el metal de colada ocurren cuando se utilizan los aceros de automoción y construcción. Los contenidos detectados para este elemento $(<0,035 \%)$ no muestran un efecto definido sobre las características estructurales de los materiales fabricados, aunque es necesario considerar otros posibles efectos no contemplados en este trabajo, como es la maquinabilidad del material ${ }^{[5]}$. Aunque se conoce la capacidad del titanio para inhibir la formación del grafito con forma esferoidal ${ }^{[5]}$, en ningún caso se han detectado malformaciones asociadas a la presencia de dicho elemento.

Otro elemento analizado en las aleaciones preparadas en este trabajo utilizando las chatarras galvanizadas procedentes del sector de automoción, es el zinc. Aunque se trata de un elemento que puede ser volatilizado en el horno durante las operaciones de fusión ${ }^{[7]}$, su contenido en los metales de colada obtenidos se encuentra en el intervalo 0,06-0,11\%. En estas condiciones, no se ha detectado ningún tipo de alteración sobre la morfología de los grafitos y/o la estructura matricial de los materiales fabricados.

Al analizar comparativamente la capacidad para originar carburos en los diferentes metales preparados, no se observan diferencias importantes. En todas las cuñas se obtienen alturas ocupadas por carburos (h) que representan el $2-3 \%$ de la altura total de este tipo de pieza test.

De este modo, es de esperar que las diferencias mostradas en la tabla $\mathrm{V}$ sean debidas a ligeras variaciones en el poder de nucleación grafítica del metal de colada y/o en las condiciones de enfriamiento. Estos resultados indican que no existe una limitación en este aspecto a la hora de emplear las chatarras seleccionadas como constituyentes de las cargas metálicas.

Las figuras 3 y 4 muestran los resultados obtenidos a partir de los ensayos mecánicos realizados sobre las piezas test. Los valores de la tensión máxima de
Tabla V. Tendencia a la formación de carburos en el metal de colada
Table V. Carbide formation tendency of the melt

\begin{tabular}{|c|c|c|}
\hline Chatarra de acero & $\mathrm{h}(\mathrm{mm})$ & 4 \\
\hline Automoción & 4,1 & $\mathbf{v}$ \\
\hline Fragmentado & 6,0 & $\varepsilon$ \\
\hline Naval & 4,0 & $\varepsilon$ \\
\hline Raíl & 5,1 & 잉 \\
\hline Raíl con alto $\mathrm{P}$ & 5,3 & \\
\hline Alimentación & 6,2 & \\
\hline Construcción & 5,5 & $30 \mathrm{~mm}$ \\
\hline
\end{tabular}

rotura (R), límite elástico (LE) y dureza aumentan con el contenido de perlita en la matriz metálica. En estas condiciones, también se produce la esperada reducción en el alargamiento (A) del material. Además del efecto provocado por la velocidad de enfriamiento, el cual puede ser fácilmente observado comparando los valores obtenidos para las tres piezas cilíndricas (Fig. 3), las propiedades mecánicas obtenidas son consecuencia de la aportación de los elementos perlitizantes por parte de las chatarras seleccionadas. Los aceros procedentes de los sectores ferroviario y, especialmente de alimentación, son los más críticos puesto que contienen manganeso, cobre y/o estaño en su composición. Este hecho abre un campo de posibilidades a la hora de optimizar los costes de proceso y rentabilizar la incorporación de estos elementos, incluyéndolos directamente en las cargas metálicas empleadas en la elaboración del metal base. Además del manganeso y cobre, el gran poder del estaño como elemento promotor de perlita origina importantes aumentos en los valores de R (Fig. 3 a)), LE (Fig. 3 b)) y la dureza (Fig. 3 d)) de las piezas test fabricadas utilizando la chatarra de alimentación. Por otra parte, es necesario indicar que no se ha detectado la presencia de carburos en estos materiales.

Al analizar comparativamente los resultados obtenidos en los bloques cúbicos (Fig. 4), se observa que el efecto descrito anteriormente para el manganeso se ve disminuido como consecuencia de las condiciones lentas de enfriamiento. Es decir, la adición de este elemento genera menores aumentos en los valores de R, LE y dureza que en el caso de la probeta normalizada y las piezas cilíndricas (comparar los metales fabricados con los aceros de automoción y naval (Fig. 3). Para el caso del alargamiento, también se obtiene una disminución más moderada. Las adiciones de cromo y vanadio en los bloques cúbi- 

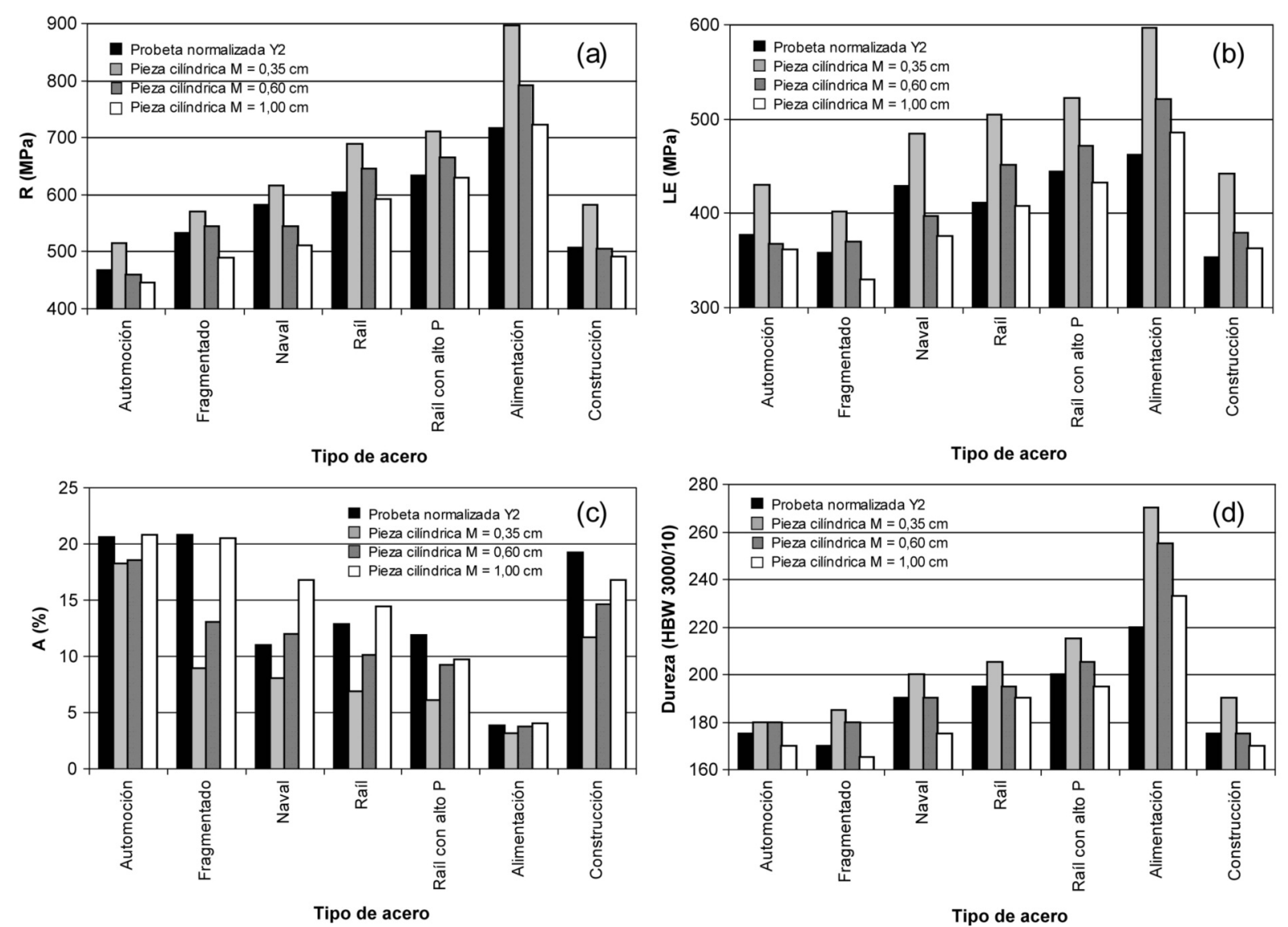

Figura 3. Propiedades mecánicas obtenidas a partir de las probetas normalizadas y las piezas cilíndricas: a) tensión de rotura $R$; b) límite elástico LE; c) alargamiento A y d) dureza.

Figure 3. Mechanical properties obtained from the standard keel-blocks and the cylindrical castings: a) tensile strength $R$; b) yield strength $L E ; c)$ elongation $A$ and d) hardness.

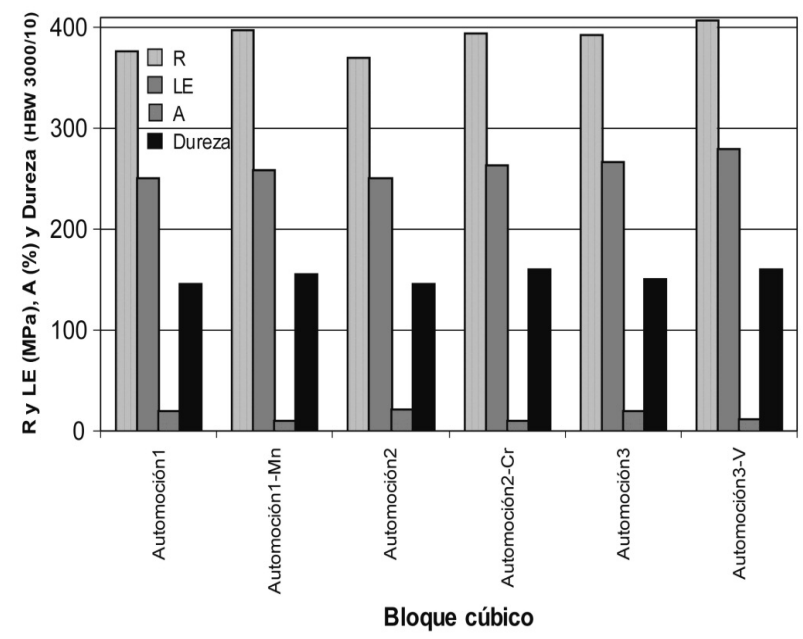

Figura 4. Propiedades mecánicas obtenidas a partir de los bloques cúbicos.

Figure 4. Mechanical properties obtained from the cubic blocks. cos dan lugar a comportamientos similares al del manganeso (Fig. 4). En ambos casos, se obtienen aumentos en los valores de R, LE y dureza de la aleación, junto con una reducción del alargamiento. Se debe indicar que la inclusión del vanadio como elemento de aleación no ha dado lugar a variaciones estructurales detectables a través de los estudios metalográficos llevados a cabo en este trabajo. Sin embargo, sus efectos afectan especialmente al alargamiento del material.

Durante el proceso destinado a la selección de las materias primas alternativas, se observó que los contenidos de manganeso y fósforo en el acero empleado para las vías férreas dependen en gran medida de su origen geográfico. Por ello, se han empleado dos tipos de chatarras constituidas por raíles con diferentes contenidos de fósforo en su composición. Tras comparar los resultados obtenidos en ambos casos, es posible comprobar que la aportación de mayores cantidades de este elemento (Tabla III) fragiliza la 
aleación fabricada, reduciendo el alargamiento y aumentando los valores de R, LE y dureza (Fig. 3).

$\mathrm{Al}$ afrontar la fabricación de piezas con matriz total o mayoritariamente ferrítica, la presencia de los elementos de aleación descritos en este trabajo se convierte en un serio inconveniente a la hora de alcanzar los requerimientos de alargamiento y/o ductilidad del material. Por ello, resulta necesario llevar a cabo estudios que permitan determinar cuál es la evolución de los contenidos de estos elementos en el metal base, mientras éste permanece fundido. Se trata de desarrollar metodologías que permitan reducir estos contenidos y ampliar la aplicación de los materiales alternativos también a la fabricación de piezas con estructuras ferríticas. Está previsto que este tipo de estudios sea objeto de futuros trabajos.

\section{CONCLUSIONES}

- La fabricación de fundición con grafito esferoidal de calidad adecuada es posible utilizando cargas metálicas que contienen hasta un $50 \%$ de diferentes chatarras de acero comerciales.

- Se han fabricado fundiciones de hierro nodular de matriz perlítica y/o ferrítico-perlítica con características estructurales y propiedades mecánicas válidas para la fabricación de piezas con requerimientos de este tipo de estructura matricial. Para ello, es necesario realizar una estimación de los elementos químicos presentes en las chatarras de acero disponibles y conocer sus implicaciones metalúrgicas.

- El elemento de aleación más importante aportado por las chatarras de acero seleccionadas en este trabajo es el manganeso. En concentraciones de hasta $0,80 \%$, este elemento ha mostrado poder ser empleado industrialmente como promotor de perlita, minimizando los costes derivados de la adición de otros elementos perlitizantes. En ningún caso se ha detectado la formación de carburos asociados a la presencia de manganeso en la matriz metálica.

- Otros elementos aportados por las chatarras de acero seleccionadas son el cobre, cromo, vanadio y estaño, siendo éste último específico de los aceros utilizados para albergar productos de alimentación. El cobre, cromo y estaño favorecen la formación de perlita en la matriz metálica y no forman carburos en las concentraciones obtenidas en este trabajo. El vanadio no muestra un efecto definido sobre las características estructurales.

- La tendencia a la formación de carburos en los metales tratados con magnesio y preparados en este trabajo no muestra variaciones importantes en función del tipo de chatarra de acero empleada para configurar la carga metálica. En este sentido, el hecho de garantizar que todos los metales preparados tengan un poder de nucleación adecuado permite la utilización de los aceros seleccionados sin riesgo de formación de carburos durante la solidificación.

- La utilización de aceros que contienen cobre, cromo y vanadio aumenta la carga de rotura y el límite elástico de las aleaciones fabricadas y disminuye el alargamiento. El efecto de los dos primeros elementos es visible en la estructura matricial resultante. Sin embargo, en el caso del vanadio no se observa diferencia alguna en los contenidos de ferrita/perlita.

- El contenido de fósforo en las chatarras de acero seleccionadas para este estudio debe ser tenido en cuenta, puesto que se trata de un elemento que fragiliza el material metálico. Salvo en el caso de determinados aceros empleados en la fabricación de raíles, la presencia de este elemento en los materiales preparados tiene su origen en componentes auxiliares adicionados habitualmente a la carga metálica (lingote de hierro, retornos y ferroaleaciones).

\section{Agradecimientos}

Los autores de este trabajo agradecen la colaboración prestada por los responsables de TQC Technologies, S.L.U., a la hora de preparar todos los materiales utilizados en este estudio y los medios facilitados para la realización de las pruebas experimentales. Este trabajo se ha realizado en el marco del proyecto MITYC 2008 (Ministerio de Industria, Turismo y Comercio de Gobierno de España), con referencia IAP-560300. 2008-66.

\section{REFERENCIAS}

[1] M. Naito, Nippon Steel Tech. Rep. Report. 94 (2006) 2-15.

[2] N. G. Bliss, AFS Trans. 105 (1997) 27-30.

[3] J. Aylen y K. Albertson, Ironmak Steelmak 33 (2006) 203-212.

[4] G.F. Geiger, W. Bauer, B.J. McKay Y P. Schumacher, Mat. Sci. Eng. A413-414 (2005) 339-345.

[5] R. A. Harding y M. L. H. Wise, AFS Trans. 108 (2000) 129-136.

[6] P. Dupin y J. M. Schissler, AFS Trans. 92 (1984) 355-360. 
[7] S. Parent-Simonin, Fonderie, (1987) 45-51.

[8] D. Venugopalan y A. Alagarsamy, AFS Trans. 98 (1990) 395-400.

[9] J. Sertucha, P. Larrañaga, J. Lacaze y M. Insausti, Int. J. Metalcast. (2010) 51-58.

[10] J. Lacaze, A. Boudot, V. Gerval, D. Oquab y H. Santos, Metall. Mater. Trans. A, 28A (1997) 2.015-2.025.

[11] R. Boeri y F. Weinberg, AFS. Trans. 97 (1989) 179-184.

[12] C. Selig y J. Lacaze, Metall. Mater. Trans. B, 31B (2000) 827-836.

[13] G.M. Goodrich, T.G. Oakwood y R.B. Gundlach, Modern Casting, (2006) 42-44.

[14] A. Loizaga, J. Sertucha y R. Suárez, An. Quím. 104 (2008) 111-119.
[15] J. Lastovica, Proc. Inter. PhD Foundry Conf. Brno, República Checa, 2009, pp 1-8.

[16] J. R. Brown, Foseco Ferrous Foundryman's Handbook Ed. Hardcover (2000) 124-125.

[17] A. Javaid y E. Essadiqi, Final report on scrap management, sorting and classification of steel (2003) Government of Canada, Report No. 2003. 23(CF).

[18] P. Larrañaga, J. Sertucha y R. Suárez, Rev. Metal Madrid 42 (2006) 244-255.

[19] F. Lietaert, Giessereiforschung 49 (1997) 106-122.

[20] E. N. Pan, M. S. Lou y C. R. Loper, AFS Trans. 95 (1987) 819-840. 